\title{
System Design for Wireless Powering of AUVs
}

\author{
Tiago Ressurreição*, Francisco Gonçalves*, Cândido Duarte*†, Ricardo Gonçalves ${ }^{\dagger}$, Rui Gomes*, \\ Rafael Santos ${ }^{\dagger}$, Ricardo Esteves ${ }^{\dagger}$, Pedro Pinto ${ }^{\dagger}$, Isabel Oliveira ${ }^{\dagger}$, and L. M. Pessoa* \\ ${ }^{*}$ INESC TEC - INESC Technology and Science \\ Campus da FEUP, Rua Dr. Roberto Frias, 4200-465 Porto, Portugal \\ ${ }^{\dagger}$ Faculty of Engineering, University of Porto \\ Rua Dr. Roberto Frias, s/n, 4200-465 Porto, Portugal \\ Email: candidoduarte@fe.up.pt, lpessoa@inesctec.pt
}

\begin{abstract}
The present work addresses the design of an electronic system for powering autonomous underwater vehicles (AUVs). We report the study and implementation of a system developed for transmitting and receiving wireless power in water from a docking station to an AUV. A simplified analysis is presented regarding the operation of a class-D power driver in a series-series inductive resonant coupling topology. We further investigate the compromise between link efficiency and power delivery of the system to circumvent the reduced power output capability. Simulation results are provided as well as measurement data validated using circuit prototypes in an experimental setup with a structure similar to the docking station of the AUV filled with salt water.
\end{abstract}

\section{INTRODUCTION}

Recently, the deployment of autonomous underwater vehicles (AUVs) has been receiving increased attention in distinct research activities [1], for instance in monitoring of deep-water rivers for environmental reasons, interaction with underwater sensor networks, ocean floor mapping for exploration of minerals, oil, gas, etc. Nevertheless, to achieve long-term and truly autonomous underwater operations, the AUV should be able to successfully recharge its batteries at an underwater docking infrastructure by means of wireless powering techniques, while avoiding any human intervention. As such, besides the demanding operational requirements for autonomous navigation, also a powerful transmitter system must be present at the docking station, whereas the AUV must comprise an efficient rectifier system and circuitry to regulate the charge rate of the batteries. Each side of the powering system should have a coupling coil, preferably the closest possible to maximize the magnetic coupling during the charging process [2], [3]. The coils need to be in a sealed housing to avoid changes in their electrical parameters along time, however this poses some limits in the minimum distance possible, which depends on the physical structures of the AUV and docking station [4]. Besides the relative distance, another inherent challenge is the proper coil alignment, which influences the impedance seen from the transmitter [5]. For that reason the system resonance can be affected, thereby having an impact on the power delivery as well as on the overall efficiency of the powering system [6].

The present work addresses the aforementioned challenges in powering an AUV. Fig. 1 depicts a physical component of the system under study, with a representation of the housings

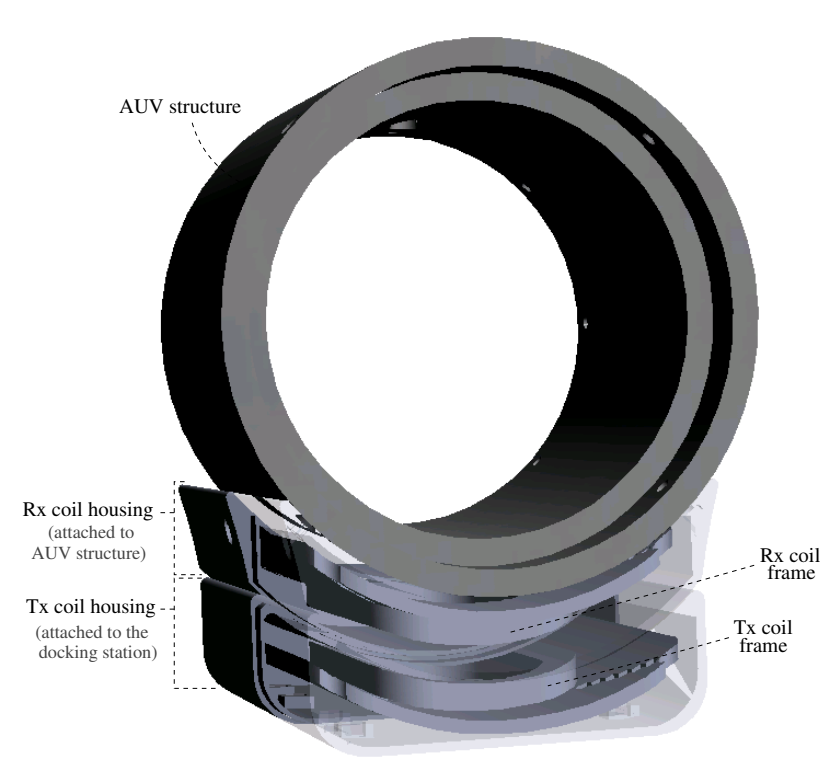

(a)

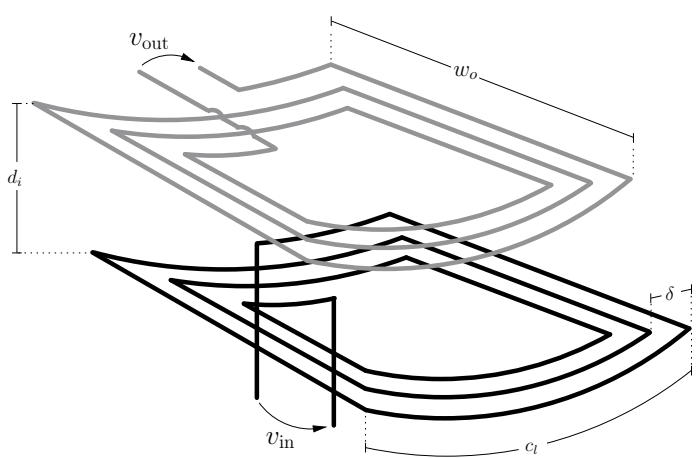

(b)

Fig. 1. (a) Coil housing structure and (b) geometry sketch.

used to incorporate the coils and a sketch of the coils employed. At the receiver side $(\mathrm{Rx})$, the coil is attached outside the AUV, minimizing the coupling distance, whereas the coil at the power transmitter (Tx) is fixed to the docking structure with a similar enclosure. The pair of coils operate as a transformer, thus, once the AUV is properly docked, the primary side (Tx) starts generating voltage at the resonance frequency 
to induce current at the secondary side, thus providing the intended power transfer through the wireless medium.

This document is organized as follows. In the next section we address the design of the coils and implementation of the power system. Section III presents the results, and sections IV and $\mathrm{V}$ are, respectively, devoted to considerations for future work and the conclusion.

\section{Wireless POWERING SySteM}

Several challenges must be overcome in designing such a system. We need to deal with losses in conductive medium due to salt water (increasing with frequency), as well as the uncertainty of the coupling factor $(k)$ due to eventual coil misalignments, which may lead to over-coupling depending on the load. In this work, we start by building the coils, characterizing them regarding the maximum efficiency and power output capability.

\section{A. Coil design}

The coupling coils are shaped according to the AUV rounded periphery, i.e. both compartments fit the $20 \mathrm{~cm} \mathrm{di-}$ ameter of the AUV cylindrical geometry [7]. In this stage, to allow the test of different coil topologies, the housings are still not filled with epoxy. Using the geometry of the coils illustrated in Fig. 1(b), we built coils with 11 turns (an equal number of turns is used in the coil pair). The gap between turns was kept the minimum possible $(\delta \rightarrow 0)$, resulting in curvature length of $\sim 19 \mathrm{~cm}$ and outer width of $\sim 20 \mathrm{~cm}-c_{l}$ and $w_{o}$ in Fig. 1(b), respectivelly. We preferred this large geometry to mitigate any eventual misalignment inherent to the docking process.

The magnet wire adopted to build the coils consists of 14AWG copper, with a $20^{\circ} \mathrm{C}$ conduction resistance of $8.4 \mathrm{~m} \Omega / \mathrm{m}$, and polyamide insulation. The self-inductance $(L)$ is practically not influenced by the water medium, i.e. it is given by the geometry and $N^{2}$. On the other hand, the equivalent series resistance $(R)$ increases with frequency due to eddy currents when the coils are separated by salt water. We extracted the electrical parameters using an impedance meter both for the air and for our underwater scenario. Fig. 2 depicts these results for $L$ and the unloaded quality factor $(Q=\omega L / R)$. As shown, the differences between air and salt water become quite evident above $100 \mathrm{kHz}$.

The "tee" model for transformers shown in Fig. 3(a) is used to implement a circuit model for the coupling coils in a seriesseries topology, where $L_{i}$ and $R_{i}$ are respectively the selfinductance and equivalent series resistance in each $i$ coil, and $M$ is the mutual inductance, $M=k \sqrt{L_{1} L_{2}}$. The capacitors are sized to establish resonance at $\omega_{0}=2 \pi f_{0}$, i.e. $\omega_{0}^{2} L_{i} C_{i}=$ 1 , where $f_{0}$ is the resonance frequency chosen - see Fig. 3(b) for an equivalent circuit when the input $V_{i}$ is a sine wave voltage at $f=f_{0}$.

The resistance $R_{21}$ depicted in Fig. 3(c) represents the equivalent resistance of the secondary reflected to the primary side, which is

$$
R_{21}=\frac{\left(\omega_{0} M\right)^{2}}{R_{2}+R_{L}}
$$

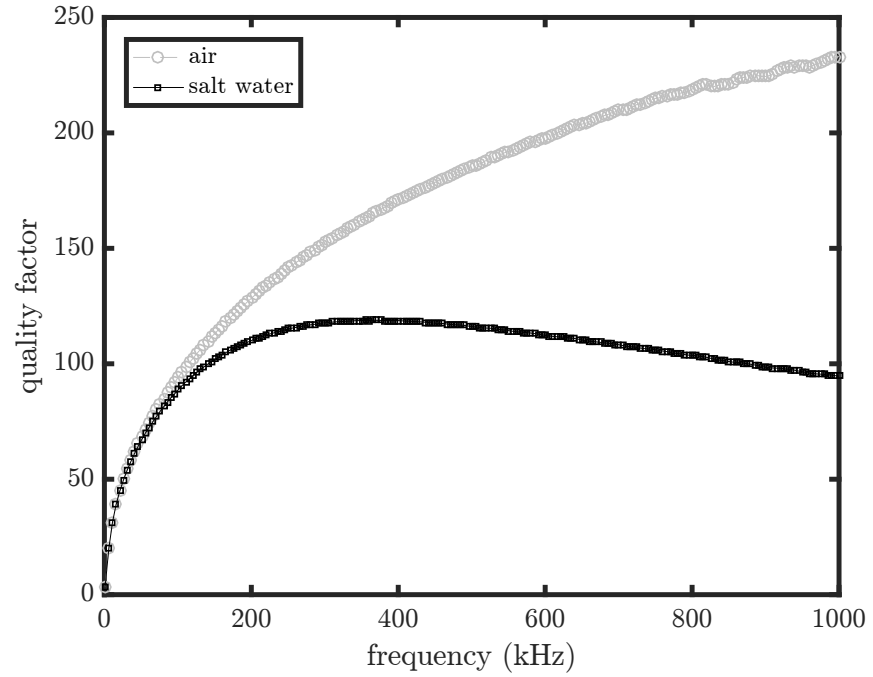

Fig. 2. $Q$ in air and salt water for $N=11$.

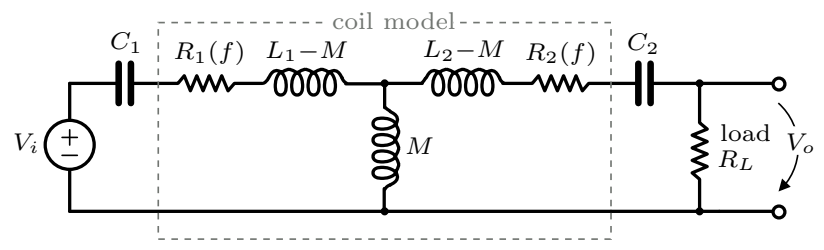

(a)

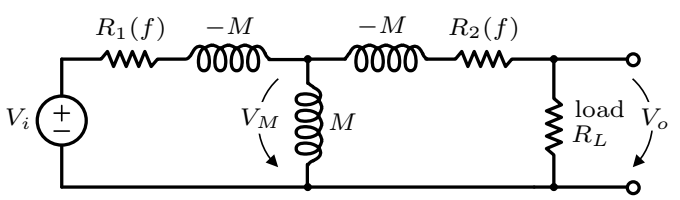

(b)

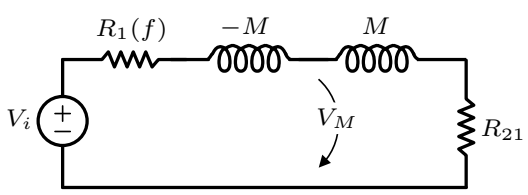

(c)

Fig. 3. (a) Electrical circuit model for the coupling system. (b) Equivalent circuit at the resonance frequency $\omega_{0}$. (c) Secondary reflected to primary side.

Hence, (1) denotes a load inversion behavior, i.e. as the load $R_{L}$ increases, it is reflected as a decrease in the input. Using the simplifications above, one can conclude that, at resonance, the power at the load $\left(P_{o}\right)$ and at the input $\left(P_{i}\right)$ are, respectively,

$$
\begin{gathered}
P_{o}=\frac{1}{2} \cdot \frac{\left(\omega_{0} M\right)^{2} R_{L}}{\left(R_{1}\left(R_{2}+R_{L}\right)+\left(\omega_{0} M\right)^{2}\right)^{2}} \cdot V_{i}^{2} \\
P_{i}=\frac{1}{2} \cdot \frac{R_{2}+R_{L}}{R_{1}\left(R_{2}+R_{L}\right)+\left(\omega_{0} M\right)^{2}} \cdot V_{i}^{2}
\end{gathered}
$$

This leads to a link efficiency $(\eta)$ given by

$$
\eta_{\text {link }}=\frac{P_{o}}{P_{i}}=\frac{\left(\omega_{0} M\right)^{2} R_{L}}{\left(R_{2}+R_{L}\right) \cdot\left(R_{1}\left(R_{2}+R_{L}\right)+\left(\omega_{0} M\right)^{2}\right)}
$$


with the following optimum load $\left(R_{L}^{\mathrm{opt}}\right)$ obtained from $\partial \eta_{\text {link }} / \partial R_{L}=0$

$$
R_{L}^{\mathrm{opt}}=R_{2} \cdot \sqrt{1+\frac{\left(\omega_{0} M\right)^{2}}{R_{1} R_{2}}}=R_{2} \cdot \sqrt{1+k^{2} Q_{1} Q_{2}}
$$

which means that, after some manipulation, the maximum attainable efficiency is

$$
\begin{aligned}
\eta_{\text {link }}^{\text {opt }} & =1+\frac{2}{k^{2} Q_{1} Q_{2}}-2 \sqrt{\frac{1}{\left(k^{2} Q_{1} Q_{2}\right)^{2}}+\frac{1}{k^{2} Q_{1} Q_{2}}} \\
& =\frac{k^{2} Q_{1} Q_{2}}{\left(1+\sqrt{1+k^{2} Q_{1} Q_{2}}\right)^{2}}
\end{aligned}
$$

Although for a fixed $k$, the efficiency can be maximized at a frequency at which $Q$ is maximum, i.e. $\eta_{\mathrm{opt}}$ at $Q_{\max }=\omega_{0} L / R$, the power output at that point may be drastically degraded. For instance, if we simply design the system to operate at $R_{L}^{\text {opt }}$, the minimum deviation on the load value can take the output power into under-coupling circumstances. Moreover a different load or resonance frequency might be preferred as the improvement on output power can be much more substantial than the efficiency drop. Fig. 4 depicts a case in which for the coils with the $Q$ given in Fig. 2(b), and assuming $k=0.25$, the frequency has been swept assuming different values for $R_{L}$. These results have been obtained through simulation of the "tee" model with the coils parameters. Cadence Spectre simulator has been used with Skill language scripting and periodic steady-state circuit analysis to sweep the different $Q$ values along the frequency.

It should be noted that the optimum load should coincide with the load at which the system operates most of the time. Hence, either the coils are designed accordingly, which is not always possible, or the frequency of operation is changed to provide better conditions. Therefore, we can define a deviation by $\alpha$ from the resonance frequency that establishes $Q_{\max }$ by choosing a capacitor $\alpha^{2}$ times lower, i.e.

$$
\varpi^{2}=\left(\alpha \omega_{0}\right)^{2}=\frac{\alpha^{2}}{L C}
$$

By choosing $R_{L}$ according to our load system and sweeping $\alpha$, one can infer about the compromise between efficiency and output power. In some applications a criterion to choose the value of $\varpi$ is the required range for the output voltage so that the load regulation can start operating - for instance to attain a minimum voltage that triggers a battery charge controller. Adopting non-optimum values for $\varpi$ (or $\alpha$ ) is justified by the very reduced power capability of this topology at typical optimum conditions. So, when there is a unlimited power source, maximizing the power output is an option if there are no device reliability issues. Fig. 5 shows the simulation results for efficiency and power output for different loads, normalized (the $x$ axis is $\alpha$ ). Each point in the figure implies resonance (the load is changed accordingly in simulation). The trade off will be always more pronounced at very low frequencies. In our application we have chosen a very low $\alpha$ (less than 10\%)

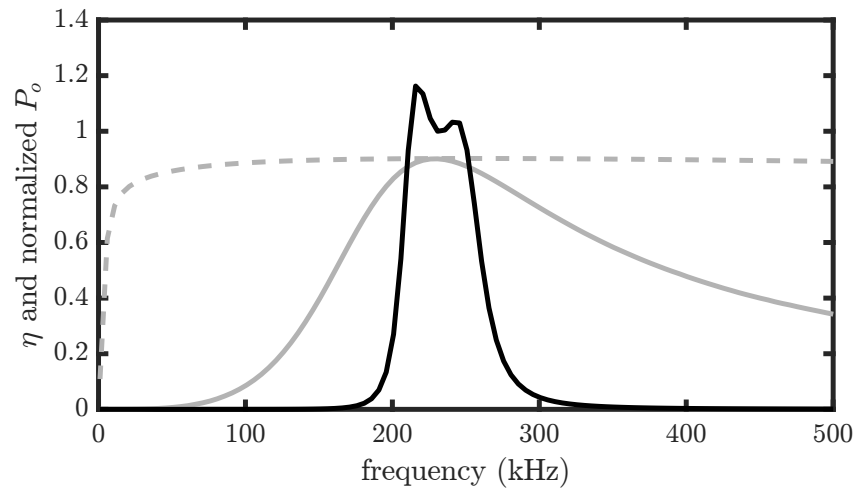

(a)

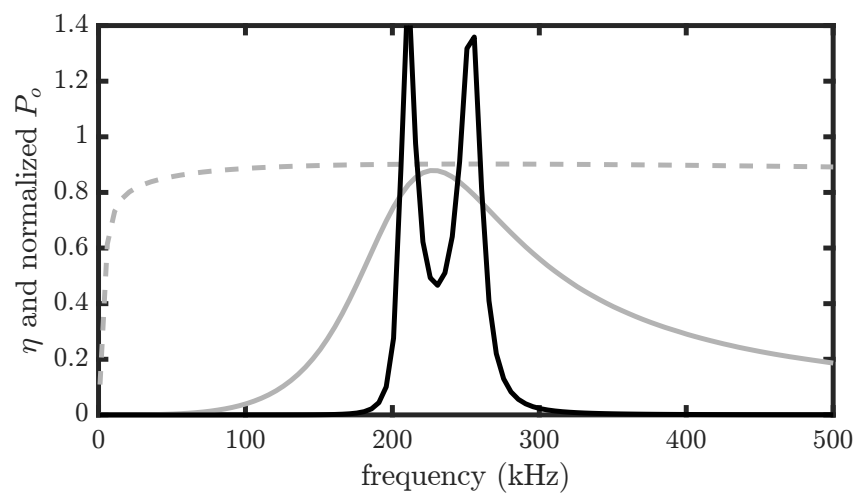

(b)

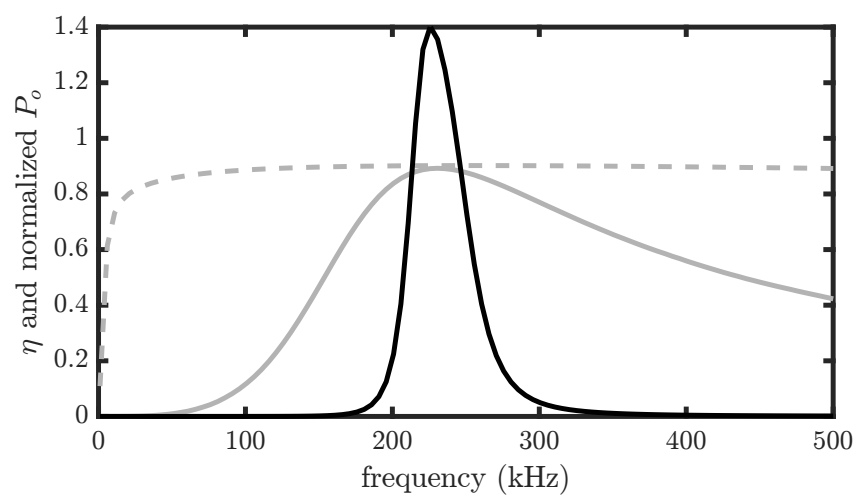

(c)

Fig. 4. Efficiency and output power for different $R_{L}$ values: (a) $R_{L}=R_{L}^{\text {opt }}$, (b) $R_{L}=0.4 R_{L}^{\mathrm{opt}}$, (c) $R_{L}=1.4 R_{L}^{\mathrm{opt}}$. The maximum theoretical efficiency for any given frequency (changing $R_{L}$ accordingly) is plotted in dashed lines. Solid gray lines denote the efficiency and black lines represent $P_{o}$ normalized to the power obtained at $R_{L}^{\text {opt }}$.

so that the secondary voltage could reach power level above $30 \mathrm{~W}$ with efficiency levels no lower than $50 \%$.

\section{B. Electronics Hardware}

The series-series topology is driven by a half-bridge class$\mathrm{D}$ inverter. If the circuit operation is defined at resonance, so that fundamental components of voltage and current into the primary coil are in phase, the resistance seen from the switching node is given by $R_{1}+R_{21}$. Let us assume a duty cycle of $50 \%$, which represents the maximum power 


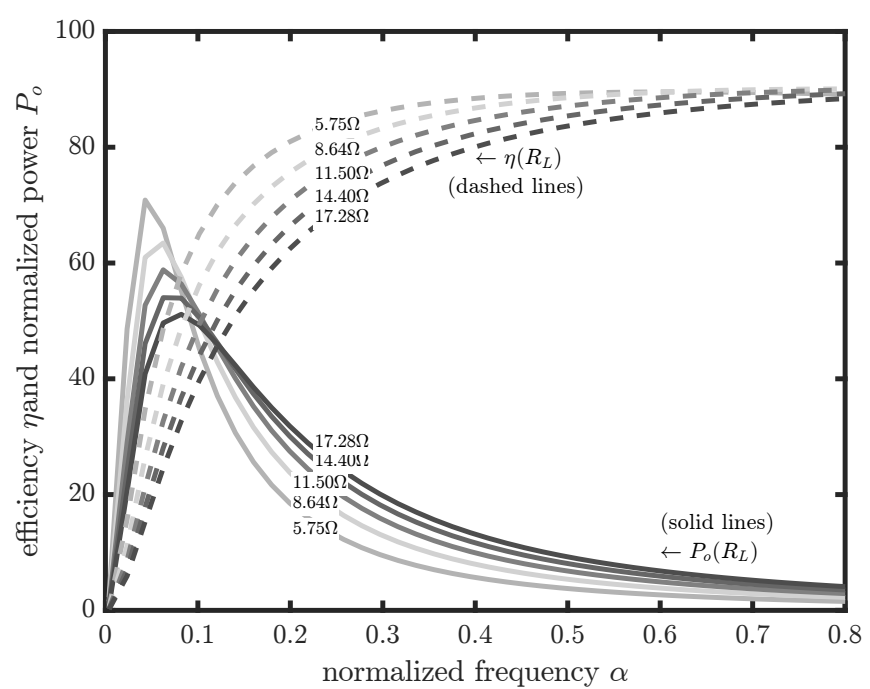

Fig. 5. Efficiency and output power for different $R_{L}$ values, $R_{L}^{\mathrm{opt}}=11.50 \Omega$. The power is normalized to the power achieved at the ideal efficiency conditions of $\eta_{\mathrm{opt}}\left(R_{L}^{\mathrm{opt}}\right)$, at the frequency denoted by $f_{\mathrm{opt}}$. The frequency axis is normalized to $f_{\text {opt }}$.

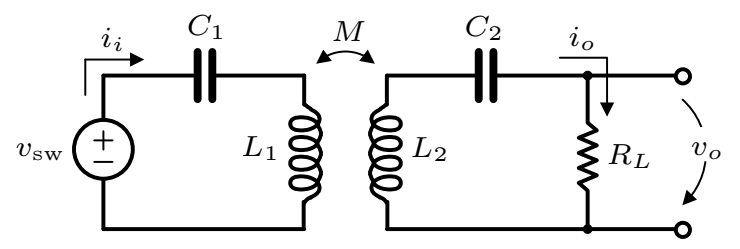

(a)

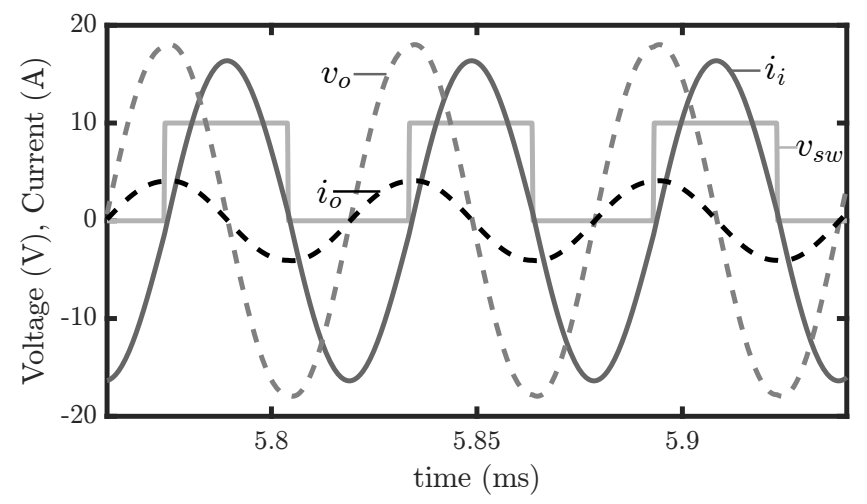

(b)

Fig. 6. (a) Circuit for ac analysis $-v_{s w}$ is a square wave voltage between 0 and $V_{D D}$, modeling the switching node of the power inverter. (b) Illustrative waveforms for the output (dashed lines) and input (solid lines) currents and voltages for the circuit in figure (a).

operation, and consider the ac operation depicted in Fig. 6(a). With a power supply of $V_{\mathrm{DD}}$ the current through the coils will be sinusoidal (i.e. a half-sine through each switch) with the magnitude determined from the fundamental component of the square waveform, i.e. $\frac{2}{\pi} V_{\mathrm{DD}}$,

$$
I_{i}=\frac{2}{\pi} \cdot \frac{V_{\mathrm{DD}}}{R_{1}+R_{21}}
$$

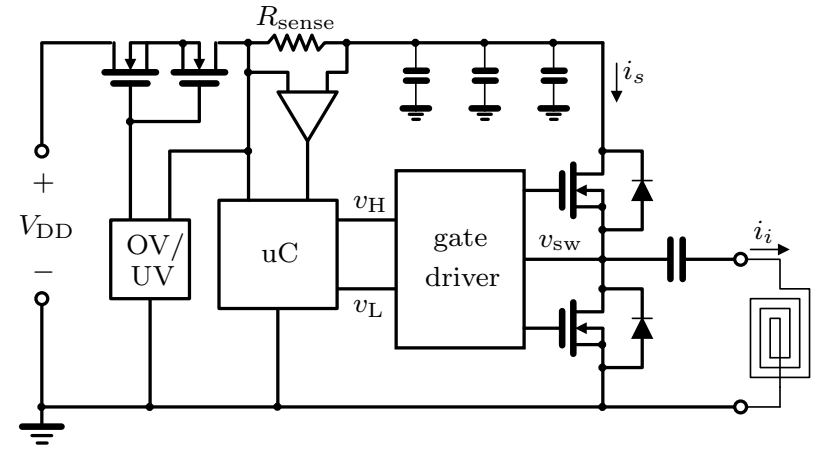

Fig. 7. Power transmitter simplified circuit diagram.

The power at the input is then

$$
P_{i}=\frac{2}{R_{1}+R_{21}} \cdot\left(\frac{V_{\mathrm{DD}}}{\pi}\right)^{2}
$$

If the switching and conduction losses are neglected at the power stage, then, based on the previous efficiency calculation in (4), the current at the output can be derived as follows

$$
I_{o}=\frac{2}{R_{1}+R_{21}} \cdot \sqrt{\frac{R_{21}}{R_{2}+R_{L}}} \cdot \frac{V_{\mathrm{DD}}}{\pi}
$$

Both (9) and (11) can be used to determine the power losses and estimate if the currents satisfy the ratings of the wires. As for the capacitors, these should handle the following ac voltage at their terminals

$$
V_{C_{1}}=I_{i} /\left(\omega_{0} C_{1}\right)
$$

and

$$
V_{C_{2}}=I_{o} /\left(\omega_{0} C_{2}\right)
$$

Some illustrative waveforms are plotted in Fig. 6(b) for the case of operation at resonance $(M=10.8 \mu \mathrm{H}, k=0.25$, $L=40 \mu \mathrm{H}, R=100 \mathrm{~m} \Omega, \omega=106 \mathrm{krad} / \mathrm{s}, R_{L}=4.4 \Omega$, $C=2.2 \mu \mathrm{F})$.

\section{Implementation}

The implementation of the power transmitter is based on the circuit with simplified representation shown in Fig. 7. The half-bridge class-D inverter makes use of two OptiMOS power N-channel MOSFETs with $8 \mathrm{~m} \Omega$ conduction resistance (BSZ060NE2LS), with a bootstrap gate driver. The ARMbased microcontroller (uC) Infineon XMC 4400 is used to monitor voltage and the current drawn from the power supply, as well as to provide pulse-width modulation (PWM) signals required for the high- and low-side MOSFETs of the inverter. The PWM signals are synthesized with fixed dead-times (around $600 \mathrm{ns)} \mathrm{to} \mathrm{minimize} \mathrm{shoot-through} \mathrm{and}$ freewheeling diode conduction [8], [9]. Power regulation is accomplished by utilizing a shunt-resistor current sensing at the input of the Tx system, including over- and under-voltage (OV/UV) protection, as well as transient suppression, and reverse voltage protection with the back-to-back MOSFETs. Both the $\mathrm{uC}$ and the current sensing circuitry are powered 


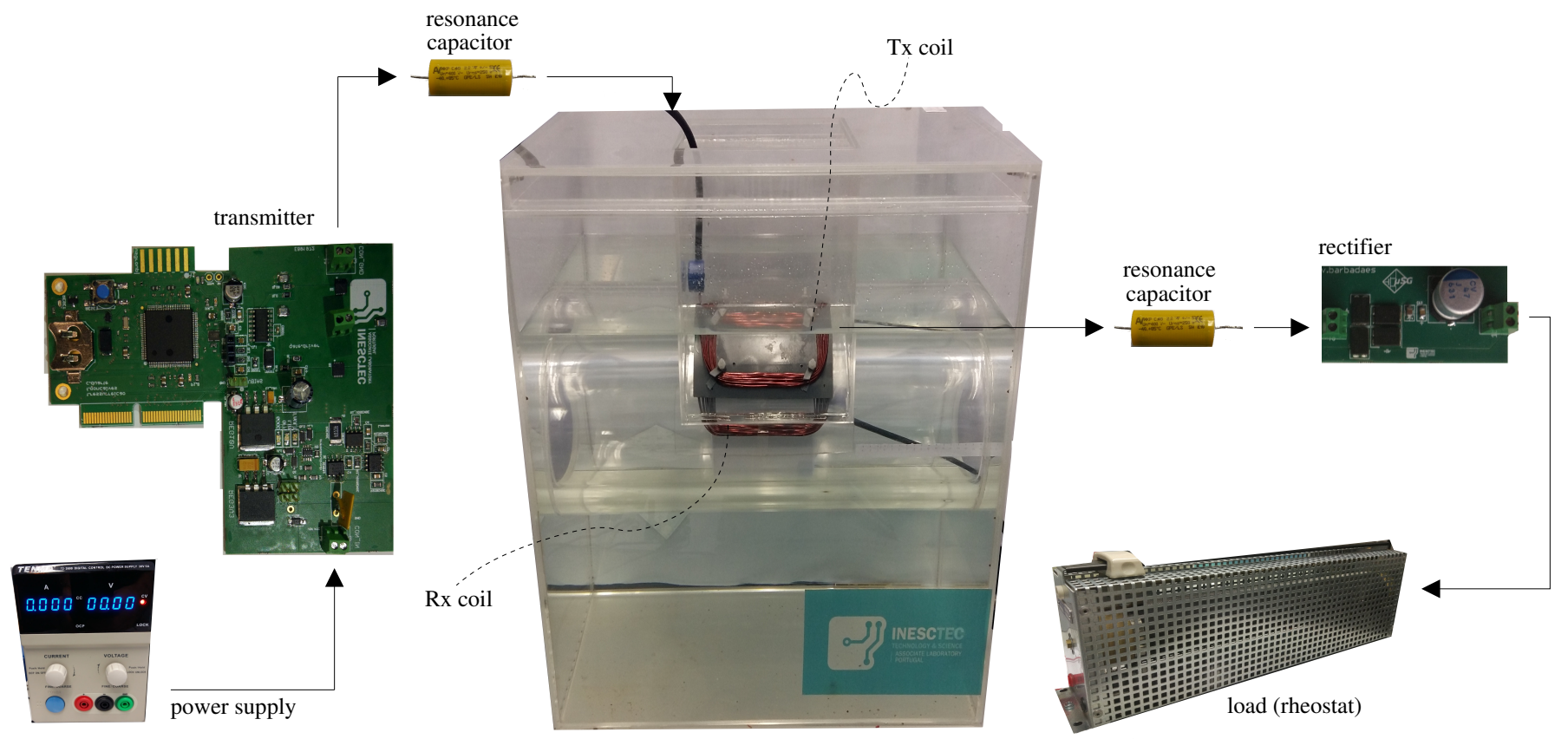

Fig. 8. Experimental setup.

by low-dropout (LDO) linear regulators, generating $10 \mathrm{~V}$ and $3.3 \mathrm{~V}$ voltage levels, with small current consumption. A webbased interface is also employed based on $\mathrm{I}^{2} \mathrm{C}$ communication between a Raspberry $\mathrm{Pi}$ and the microcontroller, to allow simple debugging and monitoring of several parameters. On the load side, full-wave rectification is employed with low forward voltage diodes in a full bridge configuration, using Schottky barrier rectifiers (PMEG045V150).

\section{RESULTS}

Fig. 8 illustrates the experimental setup used to validate the wireless power system. A container with a $20 \mathrm{~cm}$ cylindrical hole was built in $8 \mathrm{~mm}$-thick transparent acrylic, as shown in the figure - this mimics the AUV and docking station structures. In the present case, all tests were conducted with salt water with a concentration of 35 grams per liter.

The circuits for the transmitter and receiver were designed and fabricated in printed circuit boards (PCBs) with $1.55 \mathrm{~mm}$ FR-4 substrate. Coils with 11 turns have been chosen to fit the housing, adjusting it conveniently. The parameters measured for the coils used in the experiments are given in Table I together with the capacitors characterized also with the impedance meter. Based on the measurements, besides $L_{i}$ and $R_{i}$ in each $i$ coil, also the mutual inductance $M$ is obtained. As $L_{i}$ and $R_{i}$ are known, nonlinear least squares is employed with the Levenberg-Marquardt algorithm to find $k(k=0.2634)$. The coils were forced to resonate at a frequency near $17 \mathrm{kHz}$ ( $\alpha=7 \%$ ), using thin-film capacitors at both ends, with very high unloaded quality factors. As load, we used a $22 \Omega / 760 \mathrm{~W}$ rheostat, at $4.4 \Omega$. With $12 \mathrm{~V}$ power supply, we obtained a peak power of $31 \mathrm{~W}$. Fig. 9 shows the results for output voltage and efficiency, with simulation results obtained from the model
TABLE I

COIL AND RESONANCE CAPACITOR PARAMETERS OBTAINED FROM IMPEDANCE METER MEASUREMENTS.

\begin{tabular}{rl||rl|c}
\hline$R_{1}$ & 89.0 & $R_{2}$ & 78.0 & $\mathrm{~m} \Omega$ \\
$L_{1}$ & 41.020 & $L_{2}$ & 40.079 & $\mu \mathrm{H}$ \\
$C_{1}$ & 2.1747 & $C_{2}$ & 2.1847 & $\mu \mathrm{F}$ \\
$R_{c 1}$ & 7.50 & $R_{c 2}$ & 6.29 & $\mathrm{~m} \Omega$ \\
\hline
\end{tabular}

implementation in Cadence Spectre, and experimental results from practical measurements. As shown, the simulation results from the simplified model are in close agreement with the prototype measurements.

\section{FUTURE WORK}

The coil housings will be filled with an encapsulating compound (PX700K-1 from Robnor Resins). At low frequencies, it is not expected that this epoxy component will have a significant impact. In fact, due to suitable thermal characteristics, the temperature of the circuits should be kept lower than in the lab experiences because of the improved heat transfer to water. For the next designs we intend to improve significantly the power delivery, to about $70 \mathrm{~W}$, and still incorporate buckboost for output voltage regulation, so that a charge controller can be applied to ion-lithium batteries.

\section{CONClusion}

In the present work we discussed the implementation of an underwater inductive resonant powering system with the aim of being used in a docking station as a charging system for AUVs. We derived most important parameters regarding the operation of a series-series link topology with a class-D driver. The coupling coils designed have been characterized and their 


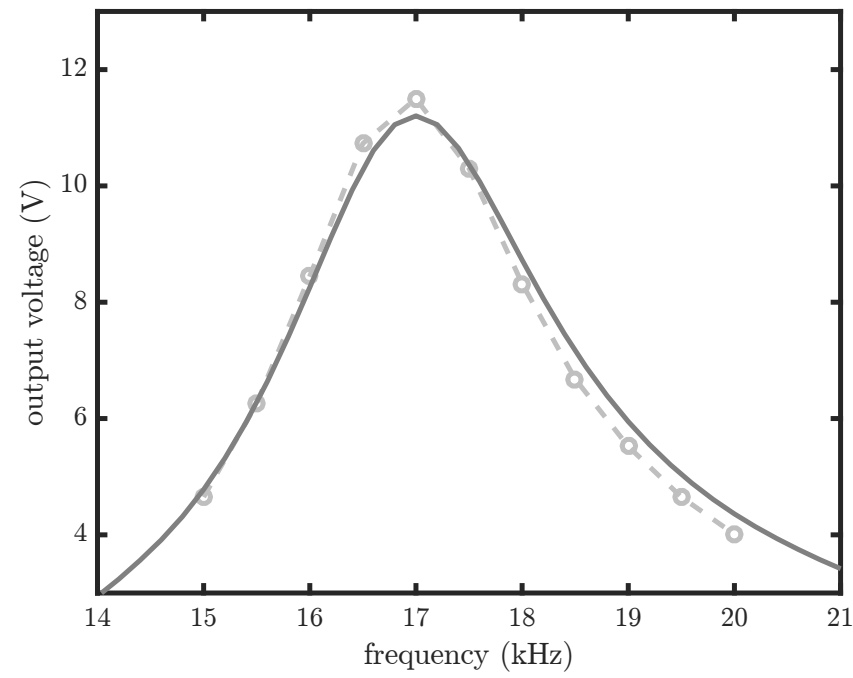

(a)

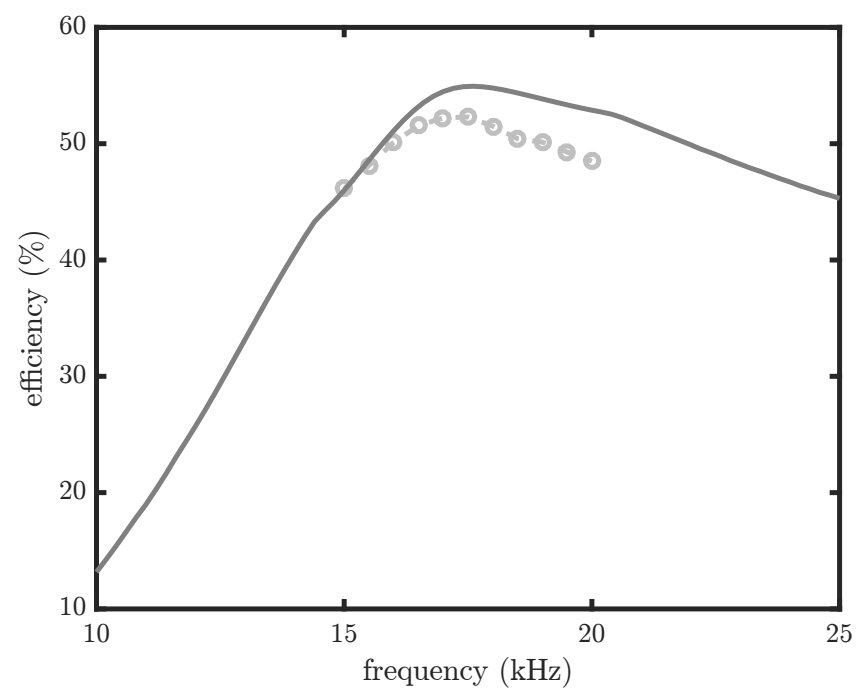

(b)

Fig. 9. (a) Output dc voltage with a load of $4.4 \Omega$ and (b) overall power efficiency.

efficiency has been analyzed. The power output and efficiency compromise has been studied and a wireless power system was designed and validated through measurements from a prototype in an experimental setup with salt water.

\section{ACKNOWLEDGMENT}

This work was supported by integrated project CORAL "NORTE-01-0145-FEDER-000036". Project NORTE-010145-FEDER-000036" is financed by the North Portugal Regional Operational Programme (NORTE 2020), under the PORTUGAL 2020 Partnership Agreement, and through the European Regional Development Fund (ERDF). The work was also carried out in the scope of project ENDURE Enabling Long-Term Deployments of Underwater Robotic Platforms in Remote Oceanic Locations (PT02 Aviso4 0015). The authors would like to thank the OceanSys group (INESC TEC), namely Rui Almeida, Bruno Ferreira, Nuno Cruz and
Aníbal Matos for all the help in the iterative definition of the structure of the coils, as well as for all the discussions regarding underwater operation.

\section{REFERENCES}

[1] J.-H. Cui, J. Kong, M. Gerla, and S. Zhou, "The challenges of building mobile underwater wireless networks for aquatic applications," IEEE Network, vol. 20, no. 3, pp. 12-18, May 2006.

[2] A. Askari, R. Stark, J. Curran, D. Rule, and K. Lin, "Underwater wireless power transfer," in IEEE Wireless Power Transfer Conference (WPTC'2015), May 2015, pp. 1-4.

[3] F. Gonçalves, A. Pereira, A. Morais, C. Duarte, R. Gomes, and L. M. Pessoa, "An adaptive system for underwater wireless power transfer," in 8th International Congress on Ultra Modern Telecommunications and Control Systems, Lisbon, Portugal, Oct 2016.

[4] J. Oiler, G. Anderson, V. Bana, A. Phipps, M. Kerber, and J. D. Rockway, "Thermal and biofouling effects on underwater wireless power transfer," in IEEE Wireless Power Transfer Conference (WPTC'2015), May 2015, pp. $1-4$.

[5] X. Huang, H. Qiang, and L. Tan, "The coil misalignment model of inductively coupled wireless power transfer system: mutual inductance analysis and transfer efficiency optimization," in Proceedings of the Progress in Electromagnetics Research Symposium, Moscow, Russia, vol. 19, 2012, p. 23.

[6] F. Gonçalves, C. Duarte, and L. M. Pessoa, "A novel circuit topology for underwater wireless power transfer," in International Conference on Systems Informatics, Modelling and Simulation, Riga, Latvia, Jun 2016, pp. $181-186$.

[7] N. A. Cruz and A. C. Matos, "The MARES AUV, a modular autonomous robot for environment sampling," in OCEANS 2008, Sep 2008, pp. 1-6.

[8] P. Amaral, C. Duarte, and P. Costa, "On the impact of timer resolution in the efficiency optimization of synchronous buck converters," International Journal of Power Electronics and Drive Systems, vol. 6, no. 4, pp. 693702, Dec 2015.

[9] A. Pereira, C. Duarte, P. Costa, and W. Gora, "Performance improvement of a buck converter using kalman filtering," International Journal of Power Electronics, vol. 8, no. 2, pp. 87-106, Mar 2017. 